\title{
SOME INEQUALITIES FOR POLYNOMIALS
}

AND RELATED ENTIRE FUNCTIONS II

Q. I. Rahman

(received January 31,1964 )

1. Let $\mathrm{p}(\mathrm{z})=\sum_{\nu=0}^{\mathrm{n}} \mathrm{a}_{\nu} z^{\nu}$ be a polynomial of degree $n$. Then clearly

(1. 1) $\int_{0}^{2 \pi}\left|P^{\prime}\left(e^{i \theta}\right)\right|^{2} d \theta=2 \pi \sum_{v=0}^{n}\left|v a_{v}\right|^{2} \leq n^{2} \int_{0}^{2 \pi}\left|P\left(e^{i \theta}\right)\right|^{2} d \theta$.

$$
\sum_{\nu=0}^{n} v\left|a_{\nu}\right|^{2} \leq n \sum_{\nu=0}^{n}\left|a_{\nu}\right|^{2}
$$

and for $R>1$

$$
\text { (1.3) } \begin{aligned}
\int_{0}^{2 \pi}\left|\mathrm{p}\left(\mathrm{Re} \mathrm{e}^{\mathrm{i} \theta}\right)\right|^{2} \mathrm{~d} \theta & =2 \pi \sum_{\nu=0}^{\mathrm{n}}\left|\mathrm{a}{ }_{\nu}\right|^{2} \mathrm{R}^{2 \nu} \leq \mathrm{R}^{2 \mathrm{n}} 2 \pi \sum_{\nu=0}^{\mathrm{n}}\left|\mathrm{a}_{\nu}\right|^{2} \\
& =\mathrm{R}^{2 \mathrm{n}} \int_{0}^{2 \pi}\left|\mathrm{p}\left(\mathrm{e}^{\mathrm{i} \theta}\right)\right|^{2} \mathrm{~d} \theta .
\end{aligned}
$$

Note that if $w=p(z)$ maps $|z|<1$ on a domain $D$ of the w-plane then the area of $D$ is given by $\pi \sum_{\nu=0}^{n} v\left|a_{\nu}\right|^{2}$.

For $p(z) \neq 0$ in $|z|<1$, inequalities (1.1) and (1.3) have been replaced respectively by the following:

Canad. Math. Bull. vol. 7, no. 4, October 1964 
(1.4) $\quad \int_{0}^{2 \pi}\left|p^{\prime}\left(e^{i \theta}\right)\right|^{2} d \theta \leq \frac{n^{2}}{2} \int_{0}^{2 \pi}\left|p\left(e^{i \theta}\right)\right|^{2} d \theta$,

(1.5) $\quad \int_{0}^{2 \pi}\left|p\left(R e^{i \theta}\right)\right|^{2} d \theta \leq \frac{R^{2 n}+1}{2} \int_{0}^{2 \pi}\left|p\left(e^{i \theta}\right)\right|^{2} d \theta, \quad(R>1)$.

Inequality (1.4) was first proved by Lax ([3], pp. 512-513; for another proof see [2], Theorem 13) and (1.5) by the author himself ([5], Theorem 1). The extremal polynomial in each case is $\mathrm{p}(\mathrm{z})=\alpha+\beta \mathrm{z}^{\mathrm{n}}$ where $|\alpha|=|\beta|$.

In this paper we shall generalize the above inequalities by considering polynomials $\mathrm{p}(\mathrm{z}) \neq 0$ in $|\mathrm{z}|<\mathrm{K}$ where $\mathrm{K}$ is an arbitrary positive number. We have not been able to solve the problem completely, e.g. we do not know the result corresponding to (1.4) when $\mathrm{p}(\mathrm{z}) \neq 0$ in $|z|<\mathrm{K}$, where $\mathrm{K}>1$.

In this connection the following result is known [4].

THEOREM A. If $p(z) \neq 0$ in $|z|<K$ where $K \geq 1$, then for $R \geq K^{2}$

(1.6)

$$
\int_{0}^{2 \pi}\left|p\left(R e^{i \theta}\right)\right|^{2} d \theta \leq \frac{R^{2 n}+K^{2 n}}{1+K^{2 n}} \int_{0}^{2 \pi}\left|p\left(e^{i \theta}\right)\right|^{2} d \theta .
$$

We prove

$$
\begin{aligned}
& \text { THEOREM 1. If } P(z)=\sum_{\nu=0}^{n} a_{\nu} z^{\nu} \text { is a polynomial of } \\
& \frac{\text { degree }}{\text { then }} \mathrm{n} \text { such that } P(z) \neq 0 \text { for }|z|<K \text {, where } K \leq 1 \text {, } \\
& \text { (1.7) } \quad \int_{0}^{2 \pi}\left|P^{\prime}\left(e^{i \theta}\right)\right|^{2} d \theta \leq \frac{n^{2}}{1+K^{2 n}} \int_{0}^{2 \pi}\left|p\left(e^{i \theta}\right)\right|^{2} d \theta,
\end{aligned}
$$


(1.8) $\sum_{v=0}^{n} v\left|a_{\nu}\right|^{2} \leq \frac{n}{1+K^{2 n}} \int_{0}^{2 \pi}\left|p\left(e^{i \theta}\right)\right|^{2} d \theta$, and for $R>1$

(1.9) $\quad \int_{0}^{2 \pi}\left|P\left(R e^{i \theta}\right)\right|^{2} d \theta \leq \frac{R^{2 n}+K^{2 n}}{1+K^{2 n}} \int_{0}^{2 \pi}\left|p\left(e^{i \theta}\right)\right|^{2} d \theta$.

$\frac{\text { In each case equality holds for }}{|\alpha|=|\beta| \text {. }} \mathrm{p}(\mathrm{z})=\alpha \mathrm{z}^{\mathrm{n}}+\beta \mathrm{K}^{\mathrm{n}}$ where

Now suppose that $\mathrm{p}(\mathrm{z})=\sum_{\nu=0}^{\mathrm{n}} \mathrm{a}_{\nu} \mathrm{z}^{\nu}, \mathrm{a}_{\mathrm{n}} \neq 0$ has all its zeros in $|z| \leq K$, where $K \geq 1$; then the polynomial $\mathrm{q}(\mathrm{z})=\mathrm{z}^{\mathrm{n}} \overline{\mathrm{p}(1 / \overline{\mathrm{z}})}=\sum_{\nu=0}^{\mathrm{n}} \overline{\mathrm{a}}_{\nu} \mathrm{z}^{\mathrm{n}-\nu}$ cannot vanish in $|\mathrm{z}|<\frac{1}{\mathrm{~K}} \leq 1$, and if we apply (1.8) to $q(z)$ we shall get

$$
\sum_{\nu=0}^{n}(n-v)\left|a{ }_{\nu}\right|^{2} \leq \frac{n K^{2 n}}{1+K^{2 n}} \sum_{\nu=0}^{n}\left|a{ }_{v}\right|^{2},
$$

or

$$
\sum_{\nu=0}^{n} v\left|a_{\nu}\right|^{2} \geq \frac{n}{1+K^{2 n}} \sum_{\nu=0}^{n}\left|a_{\nu}\right|^{2}
$$

We can therefore state the following

THEOREM 2. If $\mathrm{p}(\mathrm{z})=\sum_{\nu=0}^{\mathrm{n}} \mathrm{a}_{\nu} \mathrm{z}^{\nu}, a_{\mathrm{n}} \neq 0$ has all its zeros in $|z| \leq K$, where $K \geq 1$, then (1.10) $\sum_{\nu=0}^{\mathrm{n}} v\left|\mathrm{a}_{\nu}\right|^{2} \geq \frac{\mathrm{n}}{1+\mathrm{K}^{2 \mathrm{n}}} \sum_{\nu=0}^{\mathrm{n}}\left|\mathrm{a}_{\nu}\right|^{2}$. 
The case $K=1$ of Theorem 2 was proposed by D.J. Newman as an advanced problem in the American Mathematical Monthly (vol.69, 1962, problem No. 5040).

We can write $(1.10)$ in the equivalent form

$$
\left|\left(p^{\prime}, p\right)\right| \geq \frac{n}{1+K^{2 n}}\|p\|
$$

where $\left(p^{\prime}, p\right)$ the inner product of $p^{\prime}\left(e^{i \theta}\right)$ and $p\left(e^{i \theta}\right)$ is equal to $\left.\int_{0}^{2 \pi} p^{\prime}\left(e^{i \theta}\right) \overline{p\left(e^{i \theta}\right.}\right) d \theta$ and $\|p\|=\int_{0}^{2 \pi}\left|p\left(e^{i \theta}\right)\right|^{2} d \theta$.

Proof of (1.7). The polynomial $P(z)=p(K z)$ does not vanish for $|z|<1$ and so the polynomial $Q(z)=z^{n} \overline{P(1 / \bar{z})}$ $=z^{n} \overline{p\left(K / \frac{z}{z}\right.}$ has all its zeros in $|z| \leq 1$. Since $|Q(z)|=|P(z)|$ for $|z|=1$ it follows that $|P(z)| \leq|Q(z)|$ for $|z|>1$. From this we can conclude by a result of De Bruijn ([2], Theorem 2) that $\left|P^{\prime}(z)\right| \leq\left|Q^{\prime}(z)\right|$ for $|z| \geq 1$. In particular,

$$
\left|P^{\prime}\left(\frac{1}{K} e^{i \theta}\right)\right| \leq\left|Q^{\prime}\left(\frac{1}{K} e^{i \theta}\right)\right|
$$

for $0<K \leq 1$ and every $\theta$ such that $0 \leq \theta<2 \pi$. Now $P(z)=\sum_{v=0}^{n} a_{\nu} K^{\nu} z^{\nu}, Q(z)=\sum_{\nu=0}^{n} \bar{a}_{v} K^{\nu} z^{n-v} ;$ hence for
$0<K \leq 1$

$$
\begin{aligned}
& \int_{0}^{2 \pi}\left|P^{\prime}\left(e^{i \theta}\right)\right|^{2} d \theta=\frac{1}{K^{2}} \int_{0}^{2 \pi}\left|P^{\prime}\left(\frac{1}{K} e^{i \theta}\right)\right|^{2} d \theta \\
& \leq \frac{1}{K^{2}\left(1+K^{2 n}\right)}\left\{\int_{0}^{2 \pi}\left|P^{\prime}\left(\frac{1}{K} e^{i \theta}\right)\right|^{2} d \theta+K^{2 n} \int_{0}^{2 \pi}\left|Q^{\prime}\left(\frac{1}{K} e^{i \theta}\right)\right|^{2} d \theta\right\}
\end{aligned}
$$




$$
\begin{aligned}
& =\frac{2 \pi}{K^{2}\left(1+K^{2 n}\right)}\left\{\sum_{\nu=0}^{n} \nu^{2}\left|a{ }_{\nu}\right|^{2} K^{2}+K^{2 n} \sum_{\nu=0}^{n}(n-v)^{2}\left|a{ }_{\nu}\right|^{2} \frac{1}{K^{2 n-4 \nu-2}}\right\} \\
& =\frac{2 \pi}{1+K^{2 n}} \sum_{\nu=0}^{n}\left\{\nu^{2}+(n-v)^{2} K^{4 \nu}\right\}\left|a_{\nu}\right|^{2}
\end{aligned}
$$

$(1.13) \leq \frac{2 \pi}{1+K^{2 n}} \sum_{\nu=0}^{n}\left\{v^{2}+(n-v)^{2}\right\}\left|a{ }_{\nu}\right|^{2}$

$$
\begin{aligned}
& \leq \frac{n^{2}}{1+K^{2 n}} 2 \pi \sum_{v=0}^{n}\left|a{ }_{\nu}\right|^{2} \\
& =\frac{n^{2}}{1+K^{2 n}} \int_{0}^{2 \pi}\left|p\left(e^{i \theta}\right)\right|^{2} d \theta .
\end{aligned}
$$

Proof of (1.8). In order to prove (1.8) we start from

\section{(1.13). Thus we have}

$$
\begin{aligned}
2 \pi \sum_{\nu=0}^{n} \nu^{2}\left|a_{\nu}\right|^{2} & \leq \frac{2 \pi}{1+K^{2 n}} \sum_{\nu=0}^{n}\left\{v^{2}+(n-v)^{2}\right\}\left|a_{\nu}\right|^{2} \\
& =\frac{2 \pi}{1+K^{2 n}} \sum_{\nu=0}^{n}\left(n^{2}-2 n v+2 v^{2}\right)\left|a_{\nu}\right|^{2},
\end{aligned}
$$

or

$\frac{2 n}{1+K^{2 n}} \sum_{\nu=0}^{n} v|a|^{2} \leq \frac{n^{2}}{1+K^{2 n}} \sum_{\nu=0}^{n}\left|a_{\nu}\right|^{2}+\frac{1-K^{2 n}}{1+K^{2 n}} \sum_{\nu=0}^{n} \nu^{2}\left|a_{\nu}\right|^{2}$

$$
\leq \frac{n^{2}}{1+K^{2 n}} \sum_{\nu=0}^{n}\left|a_{\nu}\right|^{2}+\frac{1-K^{2 n}}{1+K^{2 n}} \frac{n^{2}}{1+K^{2 n}} \sum_{\nu=0}^{n}\left|a_{\nu}\right|^{2}
$$

by (1.7). Hence 


$$
\begin{aligned}
2 \mathrm{n} \sum_{v=0}^{n} v\left|a_{v}\right|^{2} & \leq n^{2} \sum_{v=0}^{n}\left|a_{v}\right|^{2}+n^{2} \frac{1-K^{2 n}}{1+K^{2 n}} \sum_{v=0}^{n}\left|a{ }_{\nu}\right|^{2} \\
& =\frac{2 n^{2}}{1+K^{2 n}} \sum_{v=0}^{n}\left|a_{v}\right|^{2}
\end{aligned}
$$

Dividing both the sides by $2 \mathrm{n}$ we get $(1,8)$.

Proof of (1.9). The polynomial $\mathrm{p}^{\prime}(\mathrm{z})$ is of degree $\mathrm{n}-1$; therefore by (1.3) we have for every $r>1$

$$
\begin{aligned}
\int_{0}^{2 \pi}\left|p^{\prime}\left(r e^{i \theta}\right)\right|^{2} d \theta & \leq r^{2 n-2} \int_{0}^{2 \pi}\left|p^{\prime}\left(e^{i \theta}\right)\right|^{2} d \theta \\
& \leq \frac{n^{2}}{1+K^{2 n}} r^{2 n-2} \int_{0}^{2 \pi}\left|p\left(e^{i \theta}\right)\right|^{2} d \theta .
\end{aligned}
$$

Multiplying both the sides by $r$ and then integrating with respect to $r$ from 1 to $\rho$ we get

$$
\sum_{\nu=0}^{n} \nu\left|a{ }_{\nu}\right|^{2}\left(\rho^{2 \nu}-1\right) \leq \frac{n}{1+K^{2 n}}\left(\rho^{2 n}-1\right) \int_{0}^{2 \pi}\left|p\left(e^{i \theta}\right)\right|^{2} d \theta .
$$

Hence for every $\rho>1$ we have

$$
\sum_{\nu=0}^{n} \nu\left|a_{\nu}\right|^{2}\left(p^{\nu}-1\right) \leq \frac{n}{1+K^{2 n}}\left(p^{n}-1\right) \int_{0}^{2 \pi}\left|p\left(e^{i \theta}\right)\right|^{2} d \theta,
$$

or

$$
\begin{aligned}
\sum_{\nu=0}^{n} \nu\left|a_{\nu}\right|^{2} \rho^{\nu} & \leq \frac{n}{1+K^{2 n}}\left(\rho^{n}-1\right) \int_{0}^{2 \pi}\left|\rho\left(e^{i \theta}\right)\right|^{2} d \theta+\sum_{\nu=0}^{n} \nu\left|a_{\nu}\right|^{2} \\
& \leq \frac{n}{1+K^{2 n}} \rho^{n} \int_{0}^{2 \pi}\left|\rho\left(e^{i \theta}\right)\right|^{2} d \theta
\end{aligned}
$$


by (1.8). Dividing both the sides of the above inequality by $P$ and integrating with respect to $\rho$ between the limits 1 and $R$ we get

$$
\sum_{\nu=0}^{n}\left|a_{\nu}\right|^{2}\left(R^{\nu}-1\right) \leq \frac{R^{n}-1}{1+K^{2 n}} \int_{0}^{2 \pi}\left|p\left(e^{i \theta}\right)\right|^{2} d \theta,
$$

or

$$
\sum_{\nu=0}^{n}\left|a{ }_{\nu}\right|^{2} R^{\nu} \leq \frac{R^{n}+K^{2 n}}{1+K^{2 n}} \int_{0}^{2 \pi}\left|p\left(e^{i \theta}\right)\right|^{2} d \theta .
$$

Finally, we replace $R$ by $R^{2}$ to get (1.9). The proof of Theorem 1 is complete.

Now the question arises as to how far the restriction $\mathrm{K} \leq 1$ is essential for the validity of estimates (1.7) and (1.8).

Let us consider the polynomial $p(z)=(z+K)^{n}$, where $K>1$. Then for arbitrarily large $\mathrm{K}$

$$
\begin{aligned}
\int_{0}^{2 \pi}\left|p\left(e^{i \theta}\right)\right|^{2} d \theta=2 \pi n^{2}\{1 & +\left({ }^{n-1} c_{1} K\right)^{2}+\ldots+\left({ }^{n-1} c_{n-2} K^{n-2}\right)^{2} \\
& \left.+\left({ }^{n-1} c_{n-1} K^{n-1}\right)^{2}\right\} \\
& \sim 2 \pi^{2} k^{2 n-2}
\end{aligned}
$$

whereas

$$
\begin{aligned}
\int_{0}^{2 \pi}\left|p\left(e^{i \theta}\right)\right|^{2} d \theta & =2 \pi\left\{1+\left({ }^{n} C_{1} K\right)^{2}+\ldots+\left({ }^{n} C_{n-1} K^{n-1}\right)^{2}+\left({ }^{n} C_{n} K^{n}\right)^{2}\right\} \\
& \sim 2 \pi K^{2 n} .
\end{aligned}
$$

Thus, for the case $K>1$, we could at the most expect to have 
(1.11) $\int_{0}^{2 \pi}\left|p^{\prime}\left(e^{i \theta}\right)\right|^{2} d \theta \leq \frac{n^{2}}{\phi(K)} \int_{0}^{2 \pi}\left|p\left(e^{i \theta}\right)\right|^{2} d \theta$,

$$
\phi(K) \sim K^{2} \text { as } K \rightarrow \infty .
$$

Such an estimate in fact holds and the proof is trivial. We simply have to note that if the zeros $z_{1}, z_{2}, \cdots, z_{n}$ of the polynomial $\mathrm{p}(\mathrm{z})=\sum_{\nu=0}^{\mathrm{n}} \mathrm{a}_{\nu} z^{\nu}$ all lie in $|z| \geq \mathrm{K}$, then on expressing $a_{\nu}$ in terms of the zeros and comparing it with $a_{0}=z_{1} z_{2} \cdots z_{n}$ we shall get

$$
\left|a_{\nu}\right| \leq\left|a_{0}\right|{ }^{n} C_{n-\nu} K^{-\nu}, \quad \nu=1,2, \ldots, n .
$$

Thus

$$
\begin{aligned}
\int_{0}^{2 \pi}\left|P^{\prime}\left(e^{i \theta}\right)\right|^{2} d \theta=2 \pi \sum_{\nu=0}^{n} \nu^{2}\left|a_{\nu}\right|^{2} & \leq 2 \pi\left|a_{0}\right|^{2} \sum_{\nu=0}^{n} \nu^{2}\left(C_{n-\nu}\right)^{2} K^{-2 \nu} \\
& \sim 2 \pi\left|a_{0}\right|^{2} n^{2} K^{-2} \text { as } K \rightarrow \infty
\end{aligned}
$$

and (1.14) follows because

$$
\int_{0}^{2 \pi}\left|p\left(e^{i \theta}\right)\right|^{2} d \theta=2 \pi \sum_{\nu=0}^{n}\left|a_{\nu}\right|^{2} \geq 2 \pi\left|a_{0}\right|^{2} .
$$

The hypothesis that the geometric mean of the moduli of the zeros is at least equal to $K$ is much weaker than the assumption $p(z) \neq 0$ in $|z|<K$. However, in this case the problem under consideration can be completely solved.

THEOREM 3. Let the geometric mean of the moduli of the zeros of a polynomial $\mathrm{p}(\mathrm{z})$ of degree $\mathrm{n}$ be $\mathrm{K}$, where $K \leq(n-1)^{-1 / 2 n}$. Then for every $R>1$. 


$$
\int_{0}^{2 \pi}\left|P\left(R e^{i \theta}\right)\right|^{2} d \theta \leq \frac{R^{2 n}+K^{2 n}}{1+K^{2 n}} \int_{0}^{2 \pi}\left|P\left(e^{i \theta}\right)\right|^{2} d \theta .
$$

If $K>(n-1)^{-1 / 2 n}$ then

$$
\int_{0}^{2 \pi}\left|p\left(R e^{i \theta}\right)\right|^{2} d \theta \leq \frac{R^{2 n}+K^{2 n}}{1+K^{2 n}} \int_{0}^{2 \pi}\left|p\left(e^{i \theta}\right)\right|^{2} d \theta,
$$

or

$$
\int_{0}^{2 \pi}\left|P\left(R e^{i \theta}\right)\right|^{2} d \theta<R^{2 n-2} \int_{0}^{2 \pi}\left|p\left(e^{i \theta}\right)\right|^{2} d \theta
$$

according as $R>R$ or $1<R<R$, where $R$ is the only $^{\top}$ root of

$$
R^{2 n}-\left(1+K^{2 n}\right) R^{2 n-2}+K^{2 n}=0
$$

in $(1, \infty)$.

\section{Proof of Theorem 3. The hypothesis implies} $\left|a_{0}\right| \geq K^{n}\left|a_{n}\right|$, so that

$$
\left|a_{n}\right|^{2} R^{2 n}+\left|a_{0}\right|^{2} \leq \frac{R^{2 n}+K^{2 n}}{1+K^{2 n}}\left(\left|a_{n}\right|^{2}+\left|a_{0}\right|^{2}\right)
$$

for $R>1$. If $1 \leq v \leq n-1$, then for $R>1$

$$
R^{2 \nu} \leq R^{2 n-2}
$$

and so

This fact follows, for example, from the Descartes' rule of signs. 


$$
\left|a_{\nu}\right|^{2} R^{2 \nu} \leq\left|a_{\nu}\right|^{2} \frac{R^{2 n}+K^{2 n}}{1+K^{2 n}}
$$

if

$$
R^{2 n-2} \leq \frac{R^{2 n}+K^{2 n}}{1+K^{2 n}}
$$

If $K \leq(n-1)^{-1 / 2 n}$ then $(1.16)$ holds for every $R>1$. Hence

$$
\begin{aligned}
\int_{0}^{2 \pi} \mid & \left.P\left(R e^{i \theta}\right)\right|^{2} d \theta=2 \pi\left\{\left(\left|a_{n}\right|^{2} R^{2 n}+\left|a_{0}\right|^{2}\right)+\left|a_{n-1}\right|^{2} R^{2 n-2}\right. \\
& \left.+\cdots+\left|a_{2}\right|^{2} R^{4}+\left|a_{1}\right|^{2} R^{2}\right\} \\
\leq & \frac{R^{2 n}+K^{2 n}}{1+K^{2 n}} 2 \pi\left\{\left(\left|a_{n}\right|^{2}+\left|a_{0}\right|^{2}\right)+\left|a_{n-1}\right|^{2}+\cdots+\left|a_{2}\right|^{2}+\left|a_{1}\right|^{2}\right\} \\
\leq & \frac{R^{2 n}+K^{2 n}}{1+K^{2 n}} \int_{0}^{2 \pi}\left|p\left(e^{i \theta}\right)\right|^{2} d \theta .
\end{aligned}
$$

But if $K>(n-1)^{-1 / 2 n}$, then for (1.16) to hold $R$ should at least be equal to $R$ where $R$ is the root of $(1.15)$ in $(1, \infty)$. If $1<R<R$ then $R^{2 n-2}$ is greater than $\frac{R^{2 n}+K^{2 n}}{1+K^{2 n}}$ as well as $\mathrm{R}^{2 v}, v=1,2, \ldots, \mathrm{n}-2$, and so we get

$$
\int_{0}^{2 \pi}\left|p\left(R e^{i \theta}\right)\right|^{2} d \theta<R^{2 n-2} \int_{0}^{2 \pi}\left|p\left(e^{i \theta}\right)\right|^{2} d \theta .
$$

Given any $\varepsilon>0$ we can clearly construct a polynomial $\mathrm{p}(\mathrm{z})=\sum_{\nu=0}^{\mathrm{n}} \mathrm{a}_{\nu} z^{\nu}$ with $\left|\mathrm{a}_{0}\right| \geq \mathrm{K}^{\mathrm{n}}\left|\mathrm{a}_{\mathrm{n}}\right|, \mathrm{K}>(\mathrm{n}-1)^{-1 / 2 \mathrm{n}}$ and 
$\int_{0}^{2 \pi}\left|p\left(R e^{i \theta}\right)\right|^{2} d \theta>(1-\varepsilon) R^{2 n-2} \int_{0}^{2 \pi}\left|p\left(e^{i \theta}\right)\right|^{2} d \theta$.

We can similarly prove the following two theorems.

THEOREM 4. Let the geometric mean of the moduli of the zeros of a polynomial $p(z)$ of degree $n$ be $K$. Then

$$
\int_{0}^{2 \pi}\left|p^{\prime}\left(e^{i \theta}\right)\right|^{2} d \theta \leq \frac{n^{2}}{1+K^{2 n}} \int_{0}^{2 \pi}\left|p\left(e^{i \theta}\right)\right|^{2} d \theta
$$

오

$$
\int_{0}^{2 \pi}\left|P^{\prime}\left(e^{i \theta}\right)\right|^{2} d \theta<(n-1)^{2} \int_{0}^{2 \pi}\left|p\left(e^{i \theta}\right)\right|^{2} d \theta
$$

according as $K \leq(2 n-1)^{1 / 2 n}(n-1)^{-1 / n}$ or $K>(2 n-1)^{1 / 2 n}(n-1)^{-1 / n}$ respectively.

THEOREM 5. If the geometric mean of the moduli of the zeros of a polynomial $p(z)=\sum_{\nu=0}^{n} a_{\nu} z^{\nu}$ of degree $n$ be $k$ then

$$
\sum_{v=0}^{n} v\left|a_{v}\right|^{2} \leq \frac{\mathrm{n}}{1+\mathrm{K}^{2 \mathrm{n}}} \int_{0}^{2 \pi}\left|\mathrm{p}\left(\mathrm{e}^{\mathrm{i \theta}}\right)\right|^{2} \mathrm{d \theta}
$$

$\underline{\text { or }}$

$$
\sum_{v=0}^{n} v\left|a_{\nu}\right|^{2}<(n-1) \int_{0}^{2 \pi}\left|p\left(e^{i \theta}\right)\right|^{2} d \theta,
$$

according as $K \leq(n-1)^{-1 / 2 n}$ or $K>(n-1)^{-1 / 2 n}$ respectively. 
2. Let $f(z)$ be an entire function of exponential type $T$, periodic on the real axis with period $2 \pi$. Then it has the form ([1], p. 109)

$$
f(z)=\sum_{\nu=-n}^{n} a_{\nu} e^{i \nu z}, \quad n \leq T
$$

In addition, if $f(z)$ is $O\left(e^{\varepsilon|z|}\right)$ on the positive imaginary axis for some $\varepsilon$ less than 1, then we shall have

$$
f(z)=\sum_{\nu=0}^{n} a_{\nu} e^{i v z}, \quad n \leq \tau
$$

Hence Theorems $A$ and 1 may be restated as follows. (We use $h_{f}(\theta)$ to denote the indicator of $f(z)$.)

THEOREM $A^{\prime}$. If $f(z) \neq 0$ for $\operatorname{Im} z>K$ where $K<0$, and if $h_{f}\left(\frac{\pi}{2}\right)<1$, then for $y<2 K$

$$
\int_{-\pi}^{\pi}|f(x+i y)|^{2} d x \leq \frac{e^{2 \tau|y|}+e^{2 \tau|K|}}{1+e^{2 \tau|K|}} \int_{-\pi}^{\pi}-|f(x)|^{2} d x .
$$

THEOREM 1'. If $f(z) \neq 0$ for $\operatorname{Im~} z>K \geq 0$, and if $h_{f}\left(\frac{\pi}{2}\right)<1$, then

$$
\begin{aligned}
& \int_{-\pi}^{\pi}\left|f^{\prime}(x)\right|^{2} d x \leq \frac{\tau^{2}}{1+e^{-2 K T}} \int_{-\pi}^{\pi}|f(x)|^{2} d x, \\
& \left|\int_{-\pi}^{\pi} f^{\prime}(x) \overline{f(x)} d x\right| \leq \frac{T}{1+e^{-2 K \tau}} \int_{-\pi}^{\pi}|f(x)|^{2} d x,
\end{aligned}
$$

and for $y<0$

$$
\int_{-\pi}^{\pi}|f(x+i y)|^{2} d x \leq \frac{e^{2 \tau|y|}+e^{-2 \tau K}}{1+e^{-2 \tau K}} \int_{-\pi}^{\pi}|f(x)|^{2} d x .
$$


For the rest of this section let $f(z)$ be an entire function of exponential type $T$ belonging to $L^{2}$ on the real axis. We shall prove results for $f(z)$ which are analogous to Theorems $A$ and 1 of the preceding section. According to a well known theorem of Paley and Wiener $([1]$, p. 103) an entire function $f(z)$ of exponential type $T$ belonging to $L^{2}$ on the real line has the representation

$$
f(z)=\int_{-T}^{T} e^{i z t} \emptyset(t) d t, \quad \emptyset L^{2} .
$$

If $\left(f^{\prime}, f\right)$ denotes the inner product of $f^{\prime}(x)$ and $f(x)$ then

$$
\left(f^{\prime}, f\right)=\int_{-\infty}^{\infty} f^{\prime}(x) \overline{f(x)} d x=2 \pi i \int_{-T}^{T} t|\varphi(t)|^{2} d t .
$$

Analogously to (1.1), (1.2) and (1.3) we have

$$
\int_{-\infty}^{\infty}\left|f^{\prime}(x)\right|^{2} d x \leq \tau^{2} \int_{-\infty}^{\infty}|f(x)|^{2} d x
$$

$$
\left|\left(f^{\prime}, f\right)\right| \leq \tau \int_{-\infty}^{\infty}|f(x)|^{2} d x
$$

and for all $y$

$$
\int_{-\infty}^{\infty}|f(x+i y)|^{2} d x \leq e^{2 \tau|y|} \int_{-\infty}^{\infty}|f(x)|^{2} d x .
$$

These inequalities follow immediately from the above representation for $f(z)$ as a finite Fourier transform.

Corresponding to (1.4) and (1.5) we have ([5], Theorems 6 and 7 ).

THEOREM B. If $f(z) \neq 0$ for $\operatorname{Im} z>0$, and if $h_{f}(\pi / 2)=0$, then 
(2.4) $\quad \int_{-\infty}^{\infty}\left|f^{\prime}(x)\right|^{2} \mathrm{~d} x \leq \frac{\tau^{2}}{2} \int_{-\infty}^{\infty}|f(x)|^{2} \mathrm{~d} x$

and for $y<0$

(2. 5)

$$
\int_{-\infty}^{\infty}|f(x+i y)|^{2} d x \leq \frac{1}{2}\left(e^{2 \tau|y|}+1\right) \int_{-\infty}^{\infty}|f(x)|^{2} d x
$$

Here we prove the following analogues of Theorem $A$ and Theorem 1 respectively.

THEOREM 6. If $f(z) \neq 0$ for Im $z>K$ where $K<0$, and if $h_{f}(\pi / 2)=0$, then for $y<2 K$

$$
\int_{-\infty}^{\infty}|f(x+i y)|^{2} d x \leq \frac{e^{2 \tau|y|}+e^{2 \tau|K|}}{1+e^{2 \tau|K|}} \int_{-\infty}^{\infty}|f(x)|^{2} d x
$$

THEOREM 7. If $f(z) \neq 0$ for $\operatorname{Im} z>K \geq 0$, and if $h_{f}(\pi / 2)=0$, then

$$
\int_{-\infty}^{\infty}\left|f^{\prime}(x)\right|^{2} d x \leq \frac{T^{2}}{1+e^{-2 K T}} \int_{-\infty}^{\infty}|f(x)|^{2} d x
$$

$$
\left|\left(f^{\prime}, f\right)\right| \leq \frac{T}{1+e^{-2 K T}} \int_{-\infty}^{\infty}|f(x)|^{2} d x
$$

and for $y<0$

$$
\int_{-\infty}^{\infty}|f(x+i y)|^{2} d x \leq \frac{e^{2 \tau|y|}+e^{-2 \tau K}}{1+e^{-2 \tau K}} \int_{-\infty}^{\infty}|f(x)|^{2} d x
$$

Let $f(z)$ be an entire function of order 1 and type $\tau$ such that $f(z)$ has all its zeros in $\operatorname{Im} z \geq K$ where $K \leq 0$. If further $h_{f}(\pi / 2)=0$ then the entire function $g(z)=e^{i \tau z \overline{f(\bar{z})}}$ has no zeros in $\operatorname{Im} z>-K \geq 0$ and $h_{g}(\pi / 2)=0$. If we apply $(2.8)$ to $g(z)$ 
we shall get

$$
\int_{0}^{T}(\tau-t)|\theta(t)|^{2} d t \leq \frac{\tau e^{-2 K T}}{1+e^{-2 K T}} \int_{0}^{\tau}|\theta(t)|^{2} d t
$$

or

$$
\int_{0}^{T} t|\phi(t)|^{2} d t \geq \frac{T}{1+e^{-2 K T}} \int_{0}^{T}|\varphi(t)|^{2} d t .
$$

We can therefore state the following analogue of Theorem 2 .

THEOREM 3. If $f(z)$ is an entire function of order 1 and type $T$ such that $f(z)$ has all its zeros in $\operatorname{Im} z \geq K$ where $\mathrm{K} \leq 0$ and $\mathrm{h}_{\mathrm{f}}(\pi / 2)=0$, then

$$
\left|\left(f^{\prime}, f\right)\right| \geq \frac{T}{1+e^{-2 K T}}\|f\|
$$

where $\|f\|=\int_{-\infty}^{\infty}|f(x)|^{2} d x$.

Proof of Theorem 6. To start with we suppose that $f(z)$ has all its zeros on Im $z=K<0$. By Paley-Wiener theorem $f(z)$ has the representation

$$
f(z)=\int_{0}^{T} e^{i z t} \phi(t) d t, \quad \phi \in L^{2} .
$$

The function $F(z)=f(z+i K)$ as well as $\omega(z)=e^{i T z} \overline{f(\bar{z}+i K)}$ has all its zeros on the real axis. Besides $|F(x)|=|\omega(x)|$ for $-\infty<x<\infty$, hence for some $\gamma$ in $0 \leq \gamma<2 \pi$ we have $F(z)=e^{i \gamma} \omega(z)$. From this it follows that

$$
|\emptyset(\tau-t)|=e^{K(\tau-2 t)}|\emptyset(t)|, \quad 0 \leq t \leq \tau
$$

Thus for $y<0$, 


$$
\begin{aligned}
\int_{-\infty}^{\infty} & |f(x+i y)|^{2} d x=2 \pi \int_{0}^{T} e^{-2 y t}|\emptyset(t)|^{2} d t \\
& =\pi \int_{0}^{T}\left[e^{-2 y t}|\varphi(t)|^{2}+e^{-2 y(\tau-t)} e^{2 K(\tau-2 t)}|\varphi(t)|^{2}\right] d t \\
& =\pi \int_{0}^{T} \frac{e^{2|K|(\tau-2 t)} e^{2|y| t}+e^{2|y|(\tau-t)}}{1+e^{2|K|(T-2 t)}}\left(\mid \varphi(t)^{2}\right. \\
& \left.+|\varphi(\tau-t)|^{2}\right) d t .
\end{aligned}
$$

Now

$$
\begin{aligned}
& \frac{e^{2 \tau|y|}+e^{2 \tau|K|}}{1+e^{2 T|K|}}-\frac{e^{2|K|(T-2 t)} e^{2|y| t}+e^{2|y|(T-t)}}{1+e^{2|K|(T-2 t)}} \\
& \quad=\frac{1}{\left(1+e^{2 \tau|K|}\right)\left(1+e^{2|K|(\tau-2 t)}\right)}\left\{( e ^ { 2 ( \tau - t ) | y | } - e ^ { 4 | K | ( \tau - t ) } ) \left(e^{2|y| t}-\right.\right. \\
& \geq 0
\end{aligned}
$$

if $y \leq 2 K$. Therefore the greatest of the quantities

$$
\frac{e^{2|K|(T-2 t)} e^{2|y| t}+e^{2|y|(\tau-t)}}{1+e^{2|K|(T-2 t)}}
$$

for $0 \leq t \leq T$ is

$$
\frac{e^{2 T|Y|}+e^{2 T|K|}}{1+e^{2 T|K|}}
$$

if $\mathrm{y} \leq 2 \mathrm{~K}$. Hence for $\mathrm{y} \leq 2 \mathrm{~K}$ we have 


$$
\int_{-\infty}^{\infty}|f(x+i y)|^{2} d x \leq \frac{e^{2 \tau|y|}+e^{2 \tau|K|}}{1+e^{2 \tau|K|}} \int_{-\infty}^{\infty}|f(x)|^{2} d x
$$

The theorem is proved for the special case when all the zeros of $f(z)$ lie on $\operatorname{Im} z=K<0$.

Now let us consider the general case. If

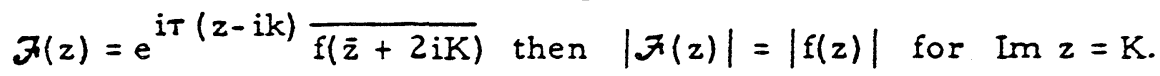
The function $G(z)=f(z+i K) e^{-i \tau z / 2}$ has no zeros for $y>0$, and $h_{G}(-\pi / 2)=h_{G}(\pi / 2)=\tau / 2$. Therefore by a theorem of Levin ([1], p. 129) we have $|G(z)| \leq|G(\bar{z})|$ for $y<0$, or $|G(z-i K)| \leq|G(\bar{z}+i K)|$ for $y<K$. Thus for $y<K$,

$$
\begin{aligned}
|f(z)| & \leq\left|f(\bar{z}+2 i K) e^{-i \tau(\bar{z}+i K) / 2}\right|\left|e^{i \tau(z-i K) / 2}\right| \\
& =\left|\overline{f(\bar{z}+2 i K)} e^{i \tau(z-i K) / 2}\right|\left|e^{i \tau(z-i K) / 2}\right| \\
& =\left|\overline{f(\bar{z}+2 i K)} e^{i \tau(z-i K)}\right| \\
& =|\mathcal{F}(z)| .
\end{aligned}
$$

Since $|G(z-i K)| \leq|G(\bar{z}+i K)|$ for $y<K$ we have

$$
|G(z+i K)| \geq|G(\bar{z}-i K)|
$$

for $\mathrm{y}>-\mathrm{K}$ or

$$
\mid G(z-i K|\geq| G(\bar{z}+i K) \mid
$$

for $y>K$. From this we can deduce in the same way as above that $\mid \mathcal{F}(z|\leq| f(z) \mid$ for $y \geq K$. Besides, it is easy to verify that for every $\eta$ such that $0 \leq \eta<2 \pi$ the function $f(z)+e^{i \eta} \mathcal{F}(z)$ has all its zeros on $\operatorname{Im} z=K$ and we can apply the special case proved above to the function $f(z)+e^{i \eta} \mathcal{F}(z)$. Hence for $y \leq 2 K$ we have 


$$
\int_{-\infty}^{\infty}\left|f(x+i y)+e^{i \eta} \mathcal{Z}(x+i y)\right|^{2} d x \leq \frac{e^{2 \tau|y|}+e^{2 \tau|K|}}{1+e^{2 \tau|K|}} \int_{-\infty}^{\infty}\left|f(x)+e^{i \eta} \mathcal{z}(x)\right|^{2} d x
$$

Now integrate both the sides with respect to $\eta$ from 0 to $2 \pi$.

On inverting the order of integration and noting the above

relations between $|f(z)|$ and $|\mathcal{F}(z)|$ for $y<K$ and $y \geq K$

we easily get

$$
\begin{aligned}
\int_{0}^{2 \pi}\left|1+e^{i \eta}\right|^{2} d \eta \int_{-\infty}^{\infty}|f(x+i y)|^{2} d x \\
\leq \frac{e^{2 \tau|y|}+e^{2 \tau|K|}}{1+e^{2 \tau|K|}} \int_{-\infty}^{\infty}|f(x)|^{2} d x \int_{0}^{2 \pi}\left|1+e^{i \eta}\right|^{2} d y .
\end{aligned}
$$

This gives the desired result.

The above estimate for $\int_{-\infty}^{\infty}|f(x+i y)|^{2} d x$ is valid only for $y \leq 2 K$. An estimate for $2 K<y<0$ can be obtained from the following consideration.

\section{Since}

$$
\int_{-\infty}^{\infty}|f(x+i y)|^{2} d x=2 \pi \int_{0}^{\tau} e^{-2 y t}|\varphi(t)|^{2} d t
$$

it is easily verified that $\log \int_{-\infty}^{\infty}|f(x+i y)|^{2} d x$ increases and is a downward convex function of $y$ as $y \rightarrow-\infty$. Thus for $2 \mathrm{~K}<\mathrm{y}<0$ we have

$$
\begin{aligned}
& \left\{\log \int_{-\infty}^{\infty}|f(x+i y)|^{2} d x-\log \int_{-\infty}^{\infty}|f(x)|^{2} d x\right\} /|y| \\
& \quad \leq\left\{\log \int_{-\infty}^{\infty}|f(x+2 i K)|^{2} d x-\log \int_{-\infty}^{\infty}|f(x+i y)|^{2} d x\right\} /(2|K|-|y|)
\end{aligned}
$$




$$
\begin{gathered}
2|K| \log \int_{-\infty}^{\infty}|f(x+i y)|^{2} d x-(2|K|-|y|) \log \int_{-\infty}^{\infty}|f(x)|^{2} d x \\
\leq|y| \log \int_{-\infty}^{\infty}|f(x+2 i K)|^{2} d x \\
\leq|y| \log \left\{\frac{e^{4 \tau|K|}+e^{2 \tau|K|}}{1+e^{2 \tau|K|}} \int_{-\infty}^{\infty}|f(x)|^{2} d x\right\}
\end{gathered}
$$

by (2.6). Hence

$2|K| \log \int_{-\infty}^{\infty}|f(x+i y)|^{2} d x \leq 2|K| \log \int_{-\infty}^{\infty}|f(x)|^{2} d x+|y| \log e^{2 T|K|}$

or

$$
\int_{-\infty}^{\infty}|f(x+i y)|^{2} d x \leq e^{\top|y|} \int_{-\infty}^{\infty}|f(x)|^{2} d x
$$

for $2 \mathrm{~K}<\mathrm{y}<0$.

Proof of (2.7). Let $F(z)=f(z+i K)$ and consider $\omega(z)=e^{i \tau z \overline{F(\bar{z})}}$, which is an entire function of exponential type $\geq \tau$. Since $f(z)$ has no zeros for $\operatorname{Im} z>K, h_{f}(\pi / 2)=0$ and $h_{f}(-\pi / 2)=T$, the function $\omega(z)$ has no zeros for Im $z<0, h_{\omega}(-\pi / 2)=T$, and $h_{\omega}(\pi / 2)=0$. The function $\mathrm{e}^{-\mathrm{i} \tau z / 2} \omega(\mathrm{z})$ therefore belongs to the class $\mathrm{P}$ discussed in ([1], p. 129) and by the theorem of Levin (loc. cit.)

$$
\left|e^{-i \tau z / 2} \omega(z)\right| \geq\left|e^{-i \tau \bar{z} / 2} \omega(\bar{z})\right|
$$

for $\operatorname{Im} z<0$. Thus for $\operatorname{Im} z<0$ we have

$$
|F(z)|=\left|e^{i \tau z \overline{\omega(\bar{z})}}\right| \leq|\omega(z)| \text {. }
$$


By another theorem of Levin $([1]$, p. 226) it follows that

$$
\left|F^{\prime}(z)\right| \leq\left|\omega^{\prime}(z)\right|
$$

for Im $z \leq 0$. In particular, $\left|F^{\prime}(x-i K)\right| \leq\left|\omega^{\prime}(x-i K)\right|$.

Since

$$
\omega(z)=e^{i \tau z} \int_{0}^{\tau} e^{-K \tau} e^{-i z t} \overline{\varphi(t)} d t
$$

we get

$$
\begin{aligned}
& \int_{-\infty}^{\infty}\left|f^{\prime}(x)\right|^{2} d x=\int_{-\infty}^{\infty}\left|F^{\prime}(x-i K)\right|^{2} d x \\
& \leq \frac{1}{1+e^{-2 K T}}\left\{\int_{-\infty}^{\infty}\left|F^{\prime}(x-i K)\right|^{2} d x+e^{-2 K T} \int_{-\infty}^{\infty} \mid \omega^{\prime}(x-i K)^{2} d x\right\} \\
& =\frac{2 \pi}{1+e^{-2 K T}}\left\{\int_{0}^{\tau} t^{2}|\varphi(t)|^{2} d t+e^{-2 K T} \int_{0}^{T}(\tau-t)^{2} e^{2 K(\tau-2 t}|\phi(t)|^{2} d t\right\} \\
& =\frac{2 \pi}{1+e^{-2 K T}} \int_{0}^{T}\left\{t^{2}+(T-t)^{2} e^{-4 K t}\right\}|\varphi(t)|^{2} d t \\
& (2.11) \leq \frac{2 \pi}{1+e^{-2 K T}} \int_{0}^{T}\left\{t^{2}+(\tau-t)^{2}\right\}|\varphi(t)|^{2} d t \\
& \leq \frac{\tau^{2}}{1+e^{-2 K T}} 2 \pi \int_{0}^{T}|\varphi(t)|^{2} d t \\
& =\frac{\tau^{2}}{1+e^{-2 K \tau}} \int_{-\infty}^{\infty}|f(x)|^{2} d x .
\end{aligned}
$$

This is (2.7) 
Proof of (2.8). From (2.11) we have

$\left.2 \pi \int_{0}^{T} t^{2}|\varphi(t)|^{2} d t \leq \frac{2 \pi}{1+e^{-2 K T}} \int_{0}^{T} \mid \tau^{2}-2 \tau t+2 t^{2}\right)|\varphi(t)|^{2} d t$

or

$\frac{2 \tau}{1+e^{-2 K T}} \int_{0}^{T} t|\theta(t)|^{2} d t \leq \frac{T^{2}}{1+e^{-2 K T}} \int_{0}^{T}|\theta(t)|^{2} d t$

$$
\begin{gathered}
+\frac{1-e^{-2 K T}}{1+e^{-2 K T}} \int_{0}^{T} t^{2}|\varphi(t)|^{2} d t \\
\leq \frac{T^{2}}{1+e^{-2 K T}} \int_{0}^{T}|\varphi(t)|^{2} d t \\
+\frac{1-e^{-2 K T}}{1+e^{-2 K T}} \frac{T^{2}}{1+e^{-2 K T}} \int_{0}^{T}|\theta(t)|^{2} d t
\end{gathered}
$$

by. (2.7). Hence

$$
\int_{0}^{T} t|\varphi(t)|^{2} d t \leq \frac{T}{1+e^{-2 K T}} \int_{0}^{T}|\varphi(t)|^{2} d t
$$

and (2. 8) follows.

Proof of (2.9). The function $f^{\prime}(z)$ is of exponential

type $T$; therefore by $(2.7)$ and (2.3) we have for every $\beta<0$

$$
\begin{aligned}
2 \pi \int_{0}^{T} t^{2} e^{-2 \beta t}|\varphi(t)|^{2} d t= & \int_{-\infty}^{\infty}\left|f^{\prime}(x+i \beta)\right|^{2} d x \\
& \leq \frac{\tau^{2}}{1+e^{-2 K T}} e^{-2 \tau \beta} \int_{-\infty}^{\infty}|f(x)|^{2} d x
\end{aligned}
$$


Integrating both the sides with respect to $\beta$ from $\delta$ to 0 we get

$2 \pi \int_{0}^{T} t|\varphi(t)|^{2}\left(e^{-2 \delta t}-1\right) d t \leq \frac{T}{1+e^{-2 K T}}\left(e^{-2 \tau \delta}-1\right) \int_{-\infty}^{\infty}|f(x)|^{2} d x$

or

$2 \pi \int_{0}^{T} t e^{-2 \delta t}|\varphi(t)|^{2} d t \leq 2 \pi \int_{0}^{T} t|\varphi(t)|^{2} d t$

$$
\begin{aligned}
& +\frac{T}{1+e^{-2 K \tau}}\left(e^{-2 \tau \delta}-1\right) \times \int_{-\infty}^{\infty}|f(x)|^{2} d x \\
& \leq \frac{\tau}{1+e^{-2 K \tau}} e^{-2 \tau \delta} \int_{-\infty}^{\infty}|f(x)|^{2} d x
\end{aligned}
$$

by (2. 8). Now integrating both the sides of the above inequality with respect to $\delta$ from $y$ to 0 we get

$2 \pi \int_{0}^{T}\left(e^{-2 y t}-1\right)|\phi(t)|^{2} d t \leq \frac{1}{1+e^{-2 K T}}\left(e^{-2 T y}-1\right) \int_{-\infty}^{\infty}|f(x)|^{2} d x$

or

$2 \pi \int_{0}^{T} e^{-2 y t}|\varphi(t)|^{2} d t \leq \frac{e^{-2 T y}+e^{-2 K T}}{1+e^{-2 K T}} \int_{-\infty}^{\infty}|f(x)|^{2} d x$

which is the same as (2.9). 


\section{REFERENCES}

1. R. P. Boas, Jr., Entire functions, Academic Press, New York 1954.

2. N. G. De Bruijn, Inequalities concerning polynomials in the complex domain, Neder. Akad. Wetensch., Proc., vol. 50 (1947), pp. 1265-1272.

3. P. D. Lax, Proof of a conjecture of P. Erdo's on the derivative of a polynomial, Bull. Amer. Math. Soc., vol. $50(1944)$, pp. 509-513.

4. M. A. Malik, An inequality for polynomials, Canadian Mathematical Bulletin, vol. 6(1963), pp. 65-69.

5. Q.I. Rahman, Some inequalities for polynomials and related entire functions, Illinois J. Math., vol. 5 (1961), pp. 144-151.

Université de Montréal

Montreal, Canada

and

Regional Engineering College

Srinagar, Kashmir, India 